\title{
Noise-robust Phase Gradient Retrieval Formulation for Phase-shifting Interferometry
}

\author{
Dae-Seo Park ${ }^{1,2}$, Beom-Hoan O $^{1,2}$, Se-Geun Park ${ }^{2}$, El-Hang Lee ${ }^{2}$, Jaehyun Park ${ }^{1}$, \\ and Seung Gol Lee ${ }^{1,2} *$ \\ ${ }^{I}$ SEM Co. - Inha University, Precision Inspection and Measurement Center (PIMC), \\ ${ }^{2}$ Optics and Photonics Elite Research Academy (OPERA), \\ Inha University, 253 Yonghyun-dong, Nam-gu, Incheon 402-751, Korea
}

(Received January 4, 2010 : revised March 31, 2010 : accepted April 5, 2010)

\begin{abstract}
Modification of the phase gradient formulation is proposed in order to make phase retrieval less susceptible to noise. The modified formulation is derived from separation of the phase terms and the intensity modulation terms of interferograms, and subsequent differentiation to reduce the noise-induced error of the phase gradient vector. Its performance is evaluated and compared to that of the conventional formulation, and noise-robust nature is confirmed.
\end{abstract}

Keywords : Phase-shifting interferometry, Phase retrieval technique, Phase gradient vector

OCIS codes : (120.0120) Instrumentation, measurement, and metrology; (100.5070) Phase retrieval;

(100.2650) Fringe analysis; (120.5050) Phase measurement

\section{INTRODUCTION}

As small-quantity batch production of electronic or optoelectronic components is widely spread in the industrial world, the importance of inspection techniques is being further emphasized, especially for versatile quality control. Most inspection techniques rely on the observation of 2-dimensional images of components. Image-based inspection is performed by a machine vision system equipped with high-resolution imaging optics and processors specialized for image processing and information extraction. Recently, more precise and reliable inspection is required, and inspection systems providing 3-dimensional shapes of small components have received much attention from industry, as the quality control of components becomes increasingly strict.

In general, 3-dimensional metrologies are categorized into three groups, contact, quasi-contact, and non-contact metrologies[1, 2]. Atomic force microscopy (AFM) and stylus are typical examples for contact metrology, and near-field scanning optical microscopy (NSOM) is a representative quasi-contact metrology application[3]. Non-contact metrology is often based on optical interferometry $[4,5]$, where the profile information is retrieved from interferograms obtained by interfering two wavefronts, one reflected from a sample under test (SUT) and the other from a reference mirror.

Among optical interferometries, phase-shifting interferometry (PSI) is the most attractive technique, due to advantages of simple configuration, automatic analysis of interferograms and immunity to variation of intensity and vibration [6-8]. In PSI, the surface profile of a SUT is obtained by phase retrieval from multiple interferograms. Several phase retrieval algorithms have been proposed and they can be categorized into two types. In the first type, the wrapped phase is found by the conventional N-Bucket method including the arctangent $\left(\tan ^{-1}\right)$ calculation, and the unwrapped phase is obtained through a complicated phase unwrapping procedure $[1,2]$. On the other hand, in the second type, the phase information is directly obtained by a line integration of the phase gradient $\nabla \phi$, where the phase gradient is usually derived from direct differentiation of cosine and sine intensity terms obtained from multiple interferograms $[9,10]$. Although the latter method does not require any complicated phase unwrapping procedures, the resultant phase can be easily deteriorated by the random occurrence of intensity noise. Since the noise reduction is one of the important issues in PSI, many attempts have been reported to reduce noise in PSI[11-18].

In this study, a modification of the phase gradient

\footnotetext{
*Corresponding author: sglee@inha.ac.kr

Color versions of one or more of the figures in this paper are available online.
} 
formulation is proposed in order to achieve noise-robust phase retrieval. Contrary to the conventional formulation, the proposed formulation is derived from separation of the phase information of each position from the interferograms, and their subsequent differentiation. In section 2, the phase retrieval techniques utilized in PSI are introduced, and the modified formulation of the phase gradient vectors is derived in section 3 . The robustness of the proposed method is evaluated for noisy interferograms in section 4, and finally its performance is discussed in section 5 .

\section{CONVENTIONAL PHASE RETRIEVAL FORMULATION BASED ON A PHASE GRADIENT VECTOR}

In PSI, an interferogram is formed by superposing the reference and the object wavefronts, and multiple interferograms required for phase retrieval can be successively obtained by introducing an additional phase $\delta_{n}$ into the reference wavefront. The interferogram can be expressed as

$$
I_{n}=I^{\prime}+I^{\prime \prime} \cos \left(\phi+\delta_{n}\right), \quad(n=0,1,2, \ldots)
$$

where $I^{\prime}, I^{\prime \prime}$ and $\phi$ are the average intensity, intensity modulation function, and phase difference between two wavefronts. In order to retrieve the phase $\phi$ from the interferograms, several phase retrieval algorithms have been proposed. For example, the 4-Bucket method requires four interferograms with different additional phases. By applying simple trigonometric identities, the equation for determining the phase difference $\phi$ can be easily derived as $[1,2]$

$$
\phi=\tan ^{-1}\left(\frac{I_{4}-I_{2}}{I_{1}-I_{3}}\right)
$$

where $I_{1}, I_{2}, I_{3}$, and $I_{4}$ are four interferograms with additional phases $\delta_{n}$ of $0, \pi / 2, \pi$, and $3 \pi / 2$, respectively. Since the phase $\phi$ calculated from Eq. (2) is limited to a range of $-\pi$ and $\pi$ due to the arctangent calculation, a phase unwrapping procedure is required for obtaining the unwrapped phase.

Contrary to the previous method, a line integration of the phase gradient $\nabla \phi$ allows us to determine the phase information directly without any phase unwrapping procedures. If the interferogram is assumed to be formed on the xy plane, the phase gradient is defined as

$$
\nabla \phi=\phi_{x} \hat{a}_{x}+\phi_{y} \hat{a}_{y},
$$

where $\phi_{x}$ and $\phi_{y}$ are the partial derivatives of the phase $\phi$, and $\hat{a}_{x}$ and $\hat{a}_{y}$ are the unit vectors along the $x$ and $y$ axes, respectively. By integrating Eq. (3) along an integral path $C$ connecting an arbitrary initial point $\left(x_{o}, y_{o}\right)$ with a specific point $(x, y)$ on the interferogram, the phase information can be determined with respect to the phase $\phi_{o}$ of an initial point.

$$
\phi-\phi_{\circ}=\int_{C} \nabla \phi \cdot d \mathbf{L}=\int_{C}\left(\phi_{x} d x+\phi_{y} d y\right)
$$

By repeatedly performing the line integration for every point placed on the interferogram, the overall phase information can be directly retrieved, and thus the 3-dimensional shape is obtained[10].

In the conventional formulation, the partial derivatives $\phi_{x}$ and $\phi_{y}$ in Eq. (4) are derived as follows[9]. By subtracting four interferograms $I_{1}, I_{2}, I_{3}$, and $I_{4}$ with each other, the cosine and sine intensities $I_{a}$ and $I_{b}$ are obtained as

$$
\begin{aligned}
& I_{a}=I_{1}-I_{3}=2 I^{\prime \prime} \cos \phi=I_{m} \cos \phi, \\
& I_{b}=I_{4}-I_{2}=2 I^{\prime \prime} \sin \phi=I_{m} \sin \phi,
\end{aligned}
$$

and

$$
I_{m}=\sqrt{I_{a}^{2}+I_{b}^{2}}=2 I^{\prime \prime},
$$

where $I_{m}$ denotes an intensity modulation. By partially differentiating Eqs. (5) and (6) with respect to $x$ and $y$, the partial derivatives of two cosine and sine intensities are obtained as

$$
\begin{aligned}
& I_{a x}=I_{m x} \cos \phi-I_{m} \sin \phi \cdot \phi_{x}, \\
& I_{a y}=I_{m y} \cos \phi-I_{m} \sin \phi \cdot \phi_{y}, \\
& I_{b x}=I_{m x} \sin \phi+I_{m} \cos \phi \cdot \phi_{x},
\end{aligned}
$$

and

$$
I_{b y}=I_{m y} \sin \phi+I_{m} \cos \phi \cdot \phi_{y},
$$

where the subscripts $x$ and $y$ denote partial derivatives with respect to $x(\partial / \partial x)$ and $y(\partial / \partial y)$, respectively. By combining Eqs. (8)-(11), the phase gradient components $\phi_{x}$ and $\phi_{y}$ are derived and expressed as

$$
\phi_{x}=\frac{I_{a} I_{b x}-I_{b} I_{a x}}{I_{a}{ }^{2}+I_{b}{ }^{2}}, \phi_{y}=\frac{I_{a} I_{b y}-I_{b} I_{a y}}{I_{a}{ }^{2}+I_{b}{ }^{2}} .
$$

In Eq. (12), $I_{a}$ and $I_{b}$ are directly given by the measured interferograms, but the partial derivatives such as $I_{a x}, I_{b x}$, $I_{a y}$, and $I_{b y}$ are approximated with the finite-differences. For example, the final finite-difference form of $\phi_{x}$ is expressed as 


$$
\begin{aligned}
\phi_{x}(x, y) \cong & \frac{I_{a}(x, y)}{\sqrt{I_{a}(x, y)^{2}+I_{b}(x, y)^{2}}} \frac{1}{2 \Delta x}\left(\frac{I_{b}(x+\Delta x, y)}{\sqrt{I_{a}(x, y)^{2}+I_{b}(x, y)^{2}}}-\frac{I_{b}(x-\Delta x, y)}{\sqrt{I_{a}(x, y)^{2}+I_{b}(x, y)^{2}}}\right) \\
& -\frac{I_{b}(x, y)}{\sqrt{I_{a}(x, y)^{2}+I_{b}(x, y)^{2}}} \frac{1}{2 \Delta x}\left(\frac{I_{a}(x+\Delta x, y)}{\sqrt{I_{a}(x, y)^{2}+I_{b}(x, y)^{2}}}-\frac{I_{a}(x-\Delta x, y)}{\sqrt{I_{a}(x, y)^{2}+I_{b}(x, y)^{2}}}\right) .
\end{aligned}
$$

Similarly, the finite-difference form of $\phi_{y}$ can be derived also.

The denominator terms in Eq. (13) are determined from the pixel intensities at the position $(x, y)$ of the measured interferograms, but the numerator terms are determined from the intensities at three different positions $(x, y)$ and $(x \pm \Delta x, y)$. Accordingly, if intensity noises are introduced randomly into pixel intensities, they will not be cancelled out or averaged out in the finitedifference formulation of the phase gradient vector. In other words, the resultant phase obtained by line integration of the phase gradient vector in Eq. (13) is inevitably susceptible to error due to noise.

\section{MODIFICATION OF THE PHASE GRADIENT FORMULATION}

In order to resolve the phase deterioration problem discussed in section 2, the conventional formulation shown in Eq. (12) is modified. The modified formulation is based on separation of the phase-related term and intensity modulation from the cosine and sine intensities. The separation is accomplished by normalizing $I_{a}$ and $I_{b}$ in Eqs. (5) and (6) with $I_{m}$, and the two phase-related terms $\Omega$ and $\Psi$ are defined as

$$
\cos \phi=\frac{I_{a}}{\sqrt{I_{a}^{2}+I_{b}^{2}}}=\Omega, \sin \phi=\frac{I_{b}}{\sqrt{I_{a}^{2}+I_{b}^{2}}}=\Psi
$$

By partially differentiating two phase-related terms in Eq. (14) with respect to $x$ and $y$, four partial derivatives are obtained as

$$
\begin{aligned}
& \frac{\partial}{\partial x} \cos \phi=-\sin \phi \cdot \phi_{x}=\Omega_{x}, \\
& \frac{\partial}{\partial y} \cos \phi=-\sin \phi \cdot \phi_{y}=\Omega_{y}, \\
& \frac{\partial}{\partial x} \sin \phi=\cos \phi \cdot \phi_{x}=\Psi_{x},
\end{aligned}
$$

and

$$
\frac{\partial}{\partial y} \sin \phi=\cos \phi \cdot \phi_{y}=\Psi_{y}
$$

Although the phase gradient components $\phi_{x}$ and $\phi_{y}$ can be directly derived from Eqs. (15)-(18), the derived components become infinite as $\sin \phi$ or $\cos \phi$ approaches zero. For elimination of this divergence, $-\sin \phi$ and $\cos \phi$ terms are multiplied to Eqs. (15) and (17), respectively, prior to derivation of the expression of $\phi_{x}$. Similarly the expression of $\phi_{y}$ can also be derived. The final expressions of $\phi_{x}$ and $\phi_{y}$ are newly formulated as

$$
\phi_{x}=\Omega \Psi_{x}-\Psi \Omega_{x}, \quad \phi_{y}=\Omega \Psi_{y}-\Psi \Omega_{y}
$$

For obtaining the finite-difference form of the phase gradient components $\phi_{x}$ and $\phi_{y}$ in Eq. (19), Eq. (14) is substituted into Eq. (19) and the partial derivatives of $\Omega$ and $\Psi$, such as $\Omega_{x}, \Psi_{x}$, $\Omega_{y}$, and $\Psi_{y}$ are approximated with centered finite-difference formula. The finite-difference form of $\phi_{x}$ in Eq. (19) at an arbitrary position $(x, y)$ can be expressed as

$$
\begin{aligned}
\phi_{x}(x, y) \cong & \frac{I_{a}(x, y)}{\sqrt{I_{a}(x, y)^{2}+I_{b}(x, y)^{2}}} \frac{1}{2 \Delta x}\left(\begin{array}{c}
\frac{I_{b}(x+\Delta x, y)}{\sqrt{I_{a}(x+\Delta x, y)^{2}+I_{b}(x+\Delta x, y)^{2}}} \\
-\frac{I_{b}(x-\Delta x, y)}{\sqrt{I_{a}(x-\Delta x, y)^{2}+I_{b}(x-\Delta x, y)^{2}}}
\end{array}\right) \\
& -\frac{I_{b}(x, y)}{\sqrt{I_{a}(x, y)^{2}+I_{b}(x, y)^{2}}} \frac{1}{2 \Delta x}\left(\begin{array}{c}
\frac{I_{a}(x+\Delta x, y)}{\sqrt{I_{a}(x+\Delta x, y)^{2}+I_{b}(x+\Delta x, y)^{2}}} \\
-\frac{I_{a}(x-\Delta x, y)}{\sqrt{I_{a}(x-\Delta x, y)^{2}+I_{b}(x-\Delta x, y)^{2}}}
\end{array}\right) .
\end{aligned}
$$

Similarly, the finite-difference form of $\phi_{y}$ can also be derived. Though other finite difference formulas such as three-point formula, five-point formula, and Richardson's extrapolation can be used for increasing the accuracy of the approximation, they often require more data points and long calculation time for processing[19].

Contrary to the conventional formulation in Eq. (13), the finite-difference form in Eq. (20) is described with the difference of two fractions determined from pixel intensities at the same points. Though $I_{a}$ and $I_{b}$ measured at three different positions of $(x, y)$ and $(x \pm \Delta x, y)$ are used, all fractional expressions in Eq. (20) are determined by equally positioned values. Accordingly, it is expected that the modified phase gradient components can reduce the error generated in the differentiation process, and therefore noise-robust phase retrieval is also expected by a noise averaging effect between measured interferograms.

\section{COMPARISON OF TWO PHASE GRADIENT FORMULATIONS}

To compare the immunization ability, the phase retrieval is simulated with a test wavefront described with a Zernike polynomial:

$$
\begin{aligned}
\phi(x, y)= & (5)+(-4.5) x \\
& +(-3)\left(1-6 y^{2}-6 x^{2}+6 y^{4}+12 x^{2} y^{2}+6 x^{4}\right),
\end{aligned}
$$

where the first term denotes the piston, the second term is the tilt about the $y$ axis, and the last term gives the 
third-order spherical astigmatism. The three dimensional shape of the test wavefront is shown in the left side of Fig. 1, where the interferogram is assumed to have a radius of 1 and spatially varying light intensity modulation $I_{m}$ with a Gaussian distribution, 123exp[-( $\left.\left(x^{2}+y^{2}\right)\right]$. When the phase step is $\pi / 2$, the four phase-shifted interferograms of a test specimen are obtained as shown in the right side of Fig. 1 and each interferogram is computed in a $256 \times$ 256 element array.

Noisy interferograms are generated by adding random intensity noises into them in order to verify the noise-robust property of the modified formulation. In phase-shifting interferometry, a lot of errors and noises can occur during capturing interferograms. They are usually caused from phase shifting errors, vibration, imperfections of interferometer, speckle, power fluctuation of a light source, dark current and so on. Since the proposed formulation is derived in order to reduce the effect of randomly occurring noises such as vibration, intensity fluctuation of a light source, and dark current, the occurrence of noises is assumed to have a Gaussian distribution. The degree of the noise is controlled by specifying the maximum noise level, defined as the percent of the maximum intensity modulation. Figure 2 shows one noisefree interferogram $I_{1}$ and two noisy ones with maximum noise levels of $20 \%$ and $50 \%$, respectively.

For three kinds of interferograms, both $\phi_{x}$ and $\phi_{y}$ are determined by using Eqs. (12) and (19), respectively, and the phase distributions are then obtained by performing the line integration in Eq. (4) and the center of the interferogram is chosen as the initial point with a zero phase value. The phase distributions obtained by the conventional formulation in Eq. (12) are shown at the top of Fig. 3, and those obtained by the modified formulation in Eq. (19) are shown in the middle. Even though greater fluctuation of the retrieved phases arises with increasing noise level, the results obtained from the modified formulation are shown
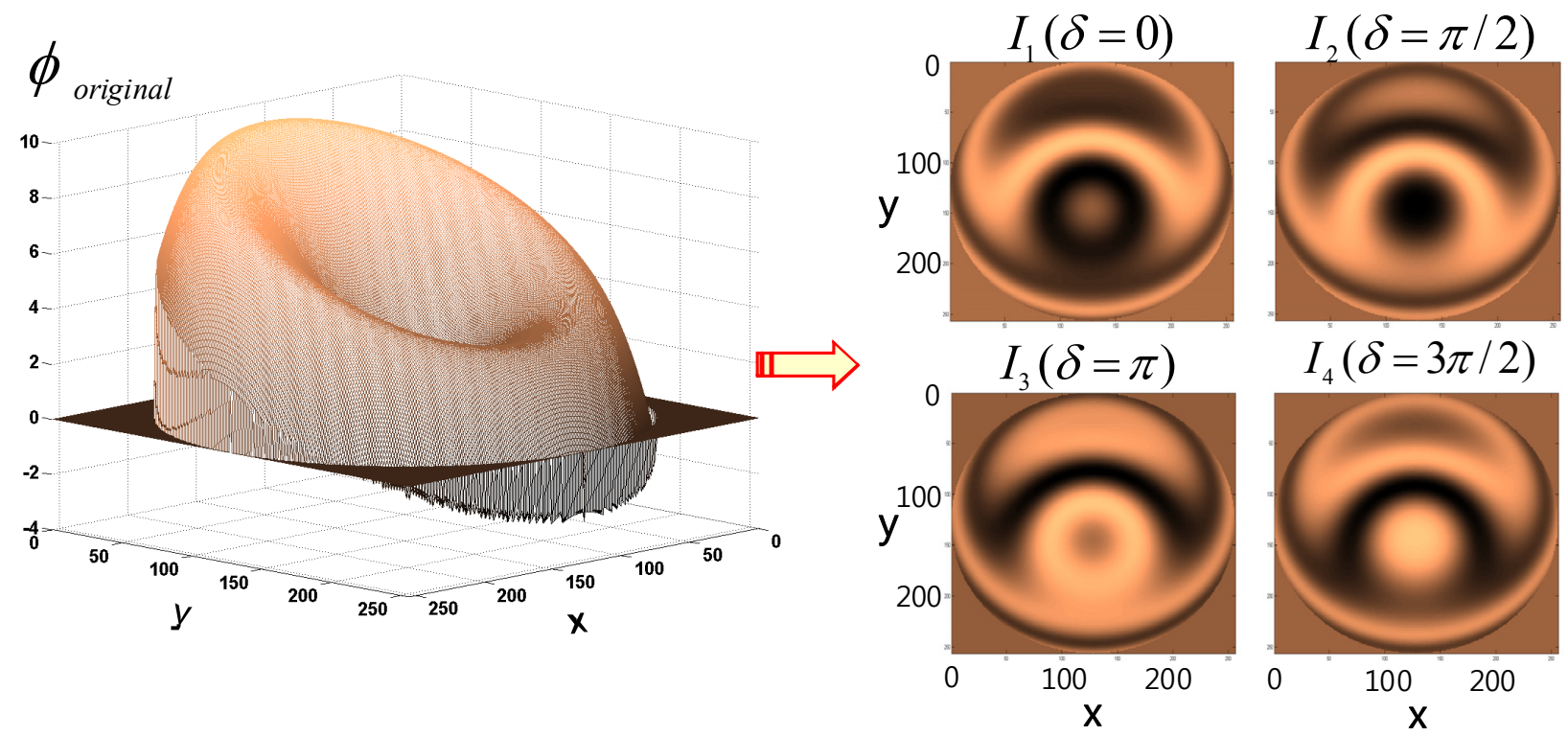

FIG. 1. The test wavefront (left) and its four phase-shifted interferograms (right).

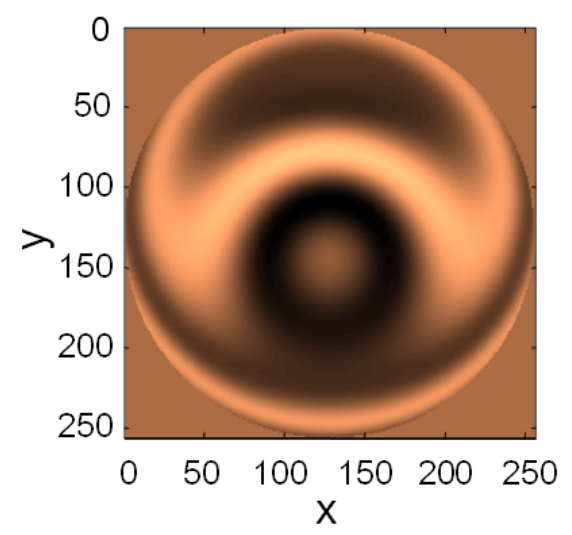

(a)

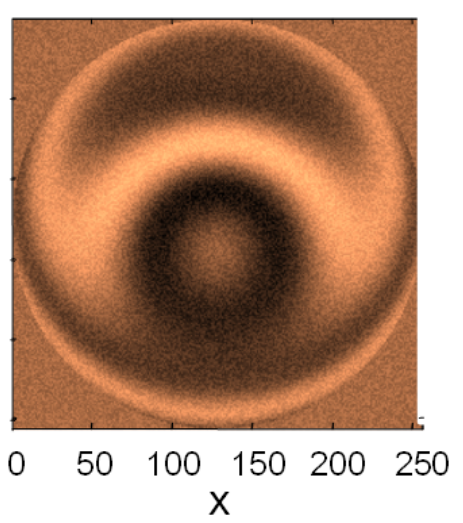

(b)

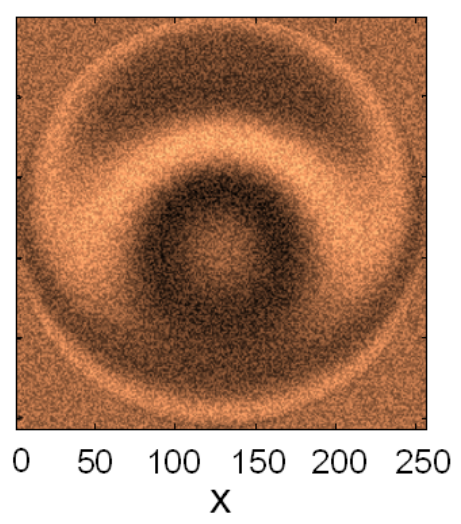

(c)

FIG. 2. Noise-free interferogram of $I_{l}$ and two noisy interferograms with the noise levels of $20 \%$ and $50 \%$, respectively. 


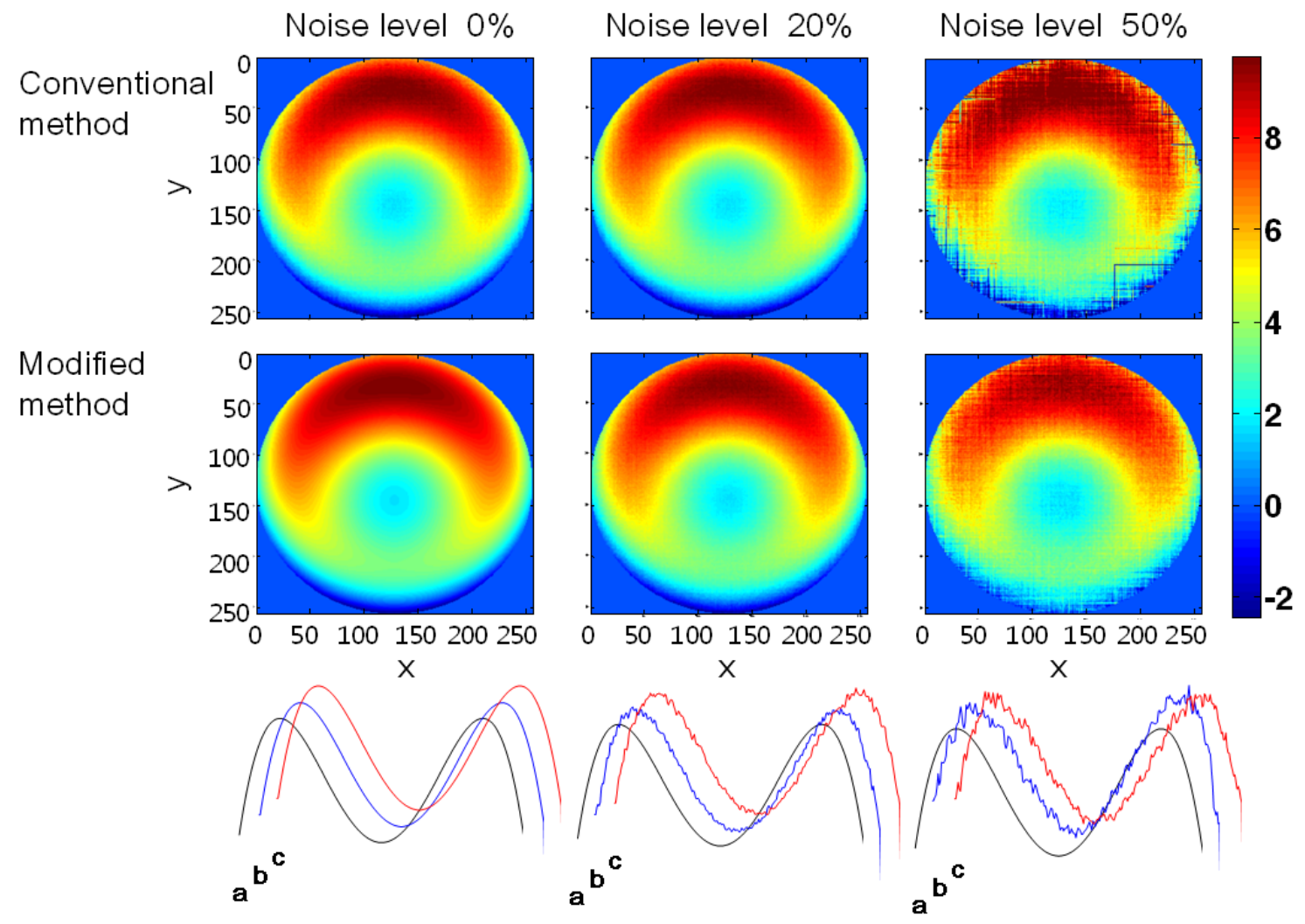

(a: reference, b: conventional method, c: modified method)

FIG. 3. Two kinds of phase distributions retrieved from the conventional formulation (top) and the modified formulation (middle) for different noise levels, and their line profiles (bottom).

to have less susceptibility to the noise. The line curves shown in the bottom side of Fig. 3 are the line profiles of the phase distributions, where a black line(a) denotes the phases of the original test wavefront, and blue(b) and red(c) lines denote the phases retrieved from the conventional and the modified formulations, respectively.

In order to compare the two formulations quantitatively, their phase deviations from the original test wavefront are evaluated, and the RMS values of the phase deviation are plotted in Fig. 4. It is clearly seen that the phase deviation of the modified formulation is relatively lower than that of the conventional formulation. Therefore, it is verified that the modified formulation for the phase gradient components offers the advantage of a noise-robust property over the conventional formulation.

Prior to determination of phase gradient terms, noisy interferograms can be preprocessed with a simple digital filter such as a $3 \times 3$ average filter. Preprocessing the interferograms with the digital filter is very useful for both the conventional and the modified formulations, because it can reduce the noise levels of noisy interferograms. However, our formulation still shows better performance than that of the conventional formulation when their performances are compared for the preprocessed interferograms with the same reduced noise level.

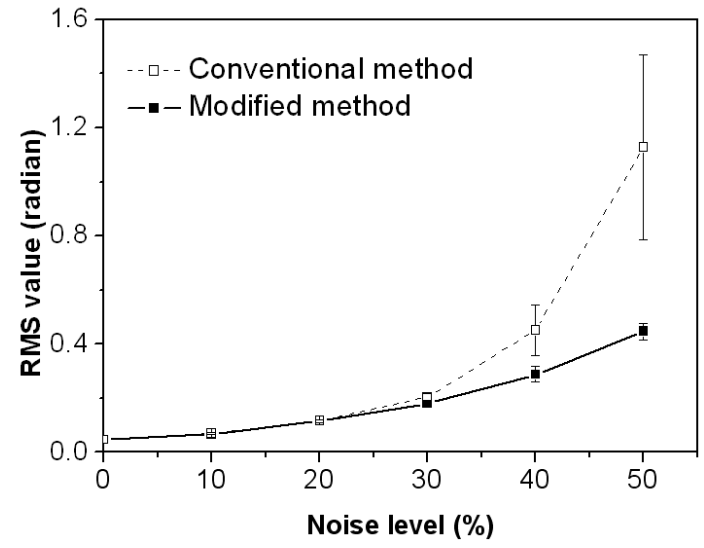

FIG. 4. RMS values of phase deviation from the test wavefront.

\section{CONCLUSION}

The formulation of the phase gradient vectors is modified for noise-robust phase retrieval, and is based on separation of the phase terms and the intensity modulation terms from the cosine and sine intensities of the measured multiple interferograms and subsequent differentiation of the phase terms. The noise-robust nature benefits from this separation, 
because random intensity noise can be partially cancelled out during the determination of the phase gradient components. The robustness of the modified formations is verified by evaluating the phase deviation from the original test wavefront and comparing the results with the deviation of the conventional formulation.

It is believed that the modified formulation will be useful even for specimens with abruptly changing profiles by carefully choosing a detoured integration path that excludes the abrupt points. For a severely noisy environment, an additional averaging algorithm or a simple digital filter may be needed to reduce the noise accumulation of the line integration.

\section{ACKNOWLEDGMENT}

This work was supported by Samsung Electro-Mechanics through the SEM Co.-Inha Univ. Precision Inspection and Measurement Center (PIMC) and the Korea Science and Engineering Foundation (KOSEF) through a Grant for the Integrated Photonics Technology Research Center (R11-2003022) at the Optics and Photonics Elite Research Academy (OPERA) at Inha University, South Korea.

\section{REFERENCES}

1. D. Malacara, Optical Shop Testing (John Wiley \& Sons Inc., New Jersey, USA, 1992).

2. D. Malacara, M. Servin, and A. Malacara, Interferogram Analysis for Optical Testing (Marcel Dekker Inc., New York, USA, 1996).

3. W. S. Ji, D. C. Kim, H. J. Kim, B. H. O, S. G. Park, E. H. Lee, and S. G. Lee, "NSOM-based characterization method applicable to optical channel waveguide with a solid-state cladding," IEEE Photon. Technol. Lett. 17, 846-848 (2005).

4. D. Kim and Y. J. Cho, "3-D surface profile measurement using an acousto-optic tunable filter based spectral phase shifting technique," J. Opt. Soc. Korea 12, 281-287 (2008).

5. T. M. Jeong, C. M. Kim, D. K. Ko, and J. Lee, "Reconstruction of wavefront aberration of 100-TW Ti:sapphire laser pulse using phase retrieval method," J. Opt. Soc. Korea 12, 186-191 (2008).

6. Y.-Y. Cheng and J. C. Wyant, "Multiple-wavelength phaseshifting interferometry," Appl. Opt. 24, 804-807 (1985).

7. R. M. Neal and J. C. Wyant, "Polarization phase-shifting point-diffraction interferometer," Appl. Opt. 45, 3463-3476 (2006).

8. S. H. Jeon and S. K. Gil, "Measurement of a mirror surface topography using 2-frame phase-shifting digital interferometry," J. Opt. Soc. Korea 13, 245-250 (2009).

9. G. Páez and M. Strojnik, "Fringe analysis and phase reconstruction from modulated intensity patterns," Opt. Lett. 22, 1669-1671 (1997).

10. G. Páez and M. Strojnik, "Phase-shifted interferometry without phase unwrapping: reconstruction of a decentered wave front," J. Opt. Soc. Am. A 16, 475-480 (1999).

11. D. S. Park, Y. S. Lee, D. C. Kim, B. H. O, S. G. Park, E. H. Lee, and S. G. Lee, "Modified formulation of phase gradient for noise-immune phase retrieval in phase-shifting interferometry," in Proc. Optical Fabrication and Testing (Rochester, NY, USA, Oct. 2008), CD, paper JWD4.

12. Y. Surrel, "Additive noise effect in digital phase detection," Appl. Opt. 36, 271-276 (1997).

13. G. Páez and M. Strojnik, "Analysis and minimization of noise effects in phase shifting interferometry," Proc. SPIE 3744, 295-305 (1999).

14. G. Páez and M. Strojnik, "Phase reconstruction from undersampled intensity patterns," J. Opt. Soc. Am. A 17, 46-52 (2000).

15. M. Servin, J. C. Estrada, J. A. Quiroga, J. F. Mosino, and M. Cywiak, "Noise in phase shifting interferometry," Opt. Exp. 17, 8789-8794 (2009).

16. P. Gao, B. Yao, N. Lindlein, K. Mantel, I. Harder, and E. Geist, "Phase-shift extraction for generalized phase-shifting interferometry," Opt. Lett. 34, 3553-3555 (2009).

17. L. L. Deck, "Suppressing phase errors from vibration in phase-shifting interferometry," Appl. Opt. 48, 3948-3960 (2009).

18. F. Bai and C. Rao, "Phase-shifts $n \pi / 2$ calibration method for phase-stepping interferometry," Opt. Exp. 17, 16861-16868 (2009).

19. R. L. Burden and J. D. Faires, Numerical Analysis (Thomson Books/Cole Publishing Company, Australia, 2005), Chapter 4. 